\title{
Der Körper als Ressource in der Sozialen Arbeit
}

\author{
Eine Rezension
}

Wendler, M./ Huster, E.-U. (Hrsg.) 2015: Der Körper als Ressource in der Sozialen Arbeit. Grundlegungen zur Selbstwirksamkeitserfahrung und Persönlichkeitsbildung. Wiesbaden: Springer VS.

Trotz seiner Relevanz und herausgehobenen Bedeutung im Kontext alltags- und lebensweltorientierter Sozialer Arbeit stellt der Körper eine, in Theorie und Praxis, noch deutlich unzureichend berücksichtigte Kategorie dar. Dieses Missverhältnis von Körpervernachlässigung in theoretischen Diskursen Sozialer Arbeit und seiner Bedeutung in der Lebenswelt der Adressatinnen und Adressaten bildet den Auftakt für die Veröffentlichung des vorliegenden Buches. Vor dem Hintergrund der gesellschaftlich gestiegenen Thematisierung des Körpers, der Etablierung der jungen Teildisziplin Körpersoziologie sowie der wegweisenden Arbeiten von Foucault zur Disziplinierung des Körpers und von Bourdieu zum habitualisierten Körper, verweisen die Herausgeber Michael Wendler und Ernst-Ulrich Huster darauf, dass die Tragweite diese bedeutsamen theoretischen Diskurse wenig Entsprechung in Form systematischer Aufarbeitungen für die Soziale Arbeit gefunden haben. In der vorliegenden Veröffentlichung wird der Körper als Ausgangspunkt konsequent lebensweltorientierter Sozialer Arbeit begriffen und in Bezug zu Handlungsfeldern der Sozialen Arbeit gesetzt.

Das Buch ist in zwei thematische Hauptabschnitte gegliedert, der erste Teil stellt relevante theoretische Positionierungen dar, der zweite Teil thematisiert die Umsetzung in die soziale Praxis.

Unter Hinzuziehung relevanter empirischer Befunde wird im theoretischen Teil zunächst von Tobias Staiger der komplexe Zusammenhang von Bewegung, körperlicher Aktivität und Sport im Verhältnis zu sozialen Faktoren wie Alter, Geschlecht, Sozialstatus und Migration einerseits, sowie zu professionellen Settings wie Frühförderung und Schule andererseits, erläutert. Angesichts deutlicher Bewegungsdisparitäten in Abhängigkeit von Herkunft, Ethnie und Geschlecht plädieren die Autoren schlussfolgernd für eine stärkere Berücksichtigung zielgruppenspezifischer Herausforderungen sowie die Bereitstellung notwendiger struktureller und finanzieller Mittel zur Förderung sozialer und körperlicher Entwicklungsmöglichkeiten.

In Rückgriff auf Bourdieu thematisieren Ernst-Ulrich Huster und Johannes D. Schütte in ihrem Beitrag „Empirische Befunde bei Bildungs- und Gesundheitsrisiken“ die besondere 
Bedeutung des Körpers bzw. des körperlichen Kapitals als konstitutives Element des Habitus. Ausgehend von der Fähigkeit zur Habitus Modifikation bzw. Habitus Transformation über die reflexive Handlung eines Menschen (Huster/Schütte: 43) plädieren die Autoren für die Bereitstellung von kindlichen Förderangeboten, die stärker Bewegungselemente und ästhetische Erziehung fokussieren. Das Vorhandensein von Kapitalaneignungsgelegenheiten im Sinne öffentlicher Förderung wird hierbei als ebenso zentral erachtet wie die individuelle Förderung von Kapitalaneignungsfähigkeiten auf der Mikroebene.

Die nun folgenden zwei Beiträge von Richard Hammer und Ernst-Ulrich Huster blicken von einer historisch-systematischen Perspektive auf den Körper bzw. auf die Geschichte des gesellschaftlichen Verhältnisses zum Körper. Beginnend mit der klassischen Antike zeichnet Richard Hammer die Entwicklung nach von der ursprünglich bestehenden untrennbaren Einheit von Körper, Geist und Seele, über die Höherstellung des Geistes in der Hochzeit der Antike bei Platon, hin zur Leibfeindlichkeit des Mittelalters (gepaart mit sozialen Praktiken exzessiver Körperlichkeit), schließlich zur Zivilisierung bzw. Disziplinierung des Körpers in der Neuzeit und zur Instrumentalisierung des Körpers in unserer Gegenwartsgesellschaft. Hammers Beitrag zeigt die wechselvolle Geschichte des Umgangs mit dem Körper, dessen untrennbare Prägung und enge Verwobenheit mit kulturellen und sozialen Paradigmen und Entwicklungen einer Epoche.

Husters Analyse fokussiert die Epoche vom 19. hin zum 20. Jahrhundert und hier insbesondere das Spannungsverhältnis von geistiger und körperlicher Entwicklung angesichts unterschiedlicher und widersprüchlicher Interessen von Wissenschaft, Politik und Praxis. Er zeigt ebenso das Entwicklungspotential wie auch das Zerstörungspotential im Umgang mit Körperlichkeit auf, kulminierend im Faschismus mit seiner sozialdarwinistischen Ideologie von Züchtung und Kampf.

In „Körper(lichkeit) in unterschiedlichen Fachdiskursen - ein Desiderat“ plädiert Michael Wendler für eine interdisziplinäre Betrachtung der dynamischen Wechselwirkung des Körpergeschehens und des psychischen Erlebens unter Nutzbarmachung aktueller Konzepte wie beispielsweise das des „Embodiments“. Dabei stellt er die besondere Bedeutung der Entwicklung des Körperkonzepts als wesentliches Fundament der Reifung des Selbstkonzepts in der kindlichen Entwicklung heraus und fordert, im Kontext von Gesundheitsförderung und Prävention, den systematischen Einbezug körperorientierter Interventionen in Kombination mit mentaler Fokussierung (Wendler: 98).

Hans-Jürgen Balz erörtert anhand von vier exemplarischen Beziehungsmustern das Verhältnis zwischen Körper und Geist in der wissenschaftlichen Psychologie. Die zentrale kommunikative Funktion des Körpers, ebenso dessen intervenierende Dimension bei psychischen Prozessen wird von ihm herausgestellt. Balz argumentiert wie Wendler auch für eine Stärkung der interdisziplinären Zusammenarbeit unter Anerkennung des Wechselverhältnisses zwischen Körper und Geist. 
Dietmar Sachser blickt auf den spielend-künstlerischen Körper aus der Perspektive der Theaterkunst. Dabei stellt er das Potential einer fundierten Reflektion szenischperformativer Vorgänge für Bildungs- und Teilhabeprozesse in der Sozialen Arbeit heraus. Den Erfahrungsmodus des „Dazwischen“ schildert er mit Rückgriff auf Henschel (2000) als wesentliches bildungstheoretisches Moment der Theaterkunst. Gleichzeitig problematisiert er die unreflektierte Funktionalisierung des Theaters bzw. des Theaterspiels als Vehikel zur Realisierung gesellschaftlicher Anpassungsmechanismen und standardisierbarer bzw. quantifizierbarer Bildung.

Den Abschluss des theoretischen Teils bildet die Befassung mit Körper, Sexualität und Geschlecht. Die Autorin Hildegard Mogge-Grotjahn erläutert die relevanten strukturtheoretischen, leibtheoretischen und Identitätsbezogenen Perspektiven auf Körper, Geschlecht und Sexualität. Für die Entwicklung professioneller Konzepte und Methoden sieht sie, neben der Notwendigkeit den professionellen Umgang mit eigenen Geschlechtskonzepten zu reflektieren, zahlreiche Anknüpfungspunkte, beispielsweise in Form von Musik-, Theater- oder Fotoprojekten.

Der zweite Abschnitt umfasst insgesamt elf Beiträge und beleuchtet die Umsetzung in die soziale Praxis aus vielfältigen, für die Soziale Arbeit relevanten, Settings und Zielgruppen. Die ersten vier Beiträge thematisieren das Thema Körper im Kontext der Erziehungsarbeit mit Kindern und Jugendlichen. Zunächst setzen sich die Autor_innen Daniele Engelbracht und Dirk Nüsken mit der hohen Bedeutung des Körpers für die Entwicklung und Förderung eines gelingenden Aufwachsens im Rahmen von Erziehungshilfe auseinander. Es folgen im Beitrag von Johannes D. Schütte die Darlegung des präventiven Empowerment-Potentials durch die Einbeziehung des Körpers bei besonders benachteiligten Kindern sowie bei Germo Zimmermann die Möglichkeit zur Ressourcenerschließung über den Körper in der offenen Kinder- und Jugendarbeit. Die Perspektive auf die Zielgruppe der Kinder und Jugendlichen wird durch die Veranschaulichung der Chancen körper- und bewegungsorientierter Arbeit im Kontext der Zirkuskunst bei Melanie Behrens und Michael Wendler abgerundet.

Die folgenden drei Beiträge befassen sich mit der Bedeutung von Körperlichkeit und Bewegung im Kontext der Sozialen Arbeit mit Erwachsenen. Nach einer grundlegenden Betrachtung der Ressource Leiblichkeit und Bewegung für die Gesunderhaltung auf körperlicher, psychischer und sozialer Ebene, stellt Ruth Haas das leiblich-seelische Schutzpotential zur Gesundheitsförderung im Spannungsverhältnis von objektiv und subjektiv empfundenen Ressourcen heraus. Marianne Eisenburger plädiert in ihrem Beitrag für die Anerkennung und Würdigung der veränderten körperlichen Bedingungen im Alter und die Berücksichtigung der weitreichenden Implikationen für das Selbstverständnis und das Selbstbild älterer Menschen, hier insbesondere im Kontext der Arbeit mit dementiell erkrankten Patienten in der Gerontopsychiatrie. Marie-Luise Hünerbein thematisiert die Rolle des professionellen Körpers in der Frühförderung im 
Rahmen (heil-)pädagogischen Handelns in begleitenden Prozessen mit Eltern. Tonischemotionale Resonanzfähigkeit wird von ihr als zentrale Ressource für die Bereitschaft zur Selbstreflexion sowie für die professionelle Weiterentwicklung angesehen.

Der Abschnitt zur Umsetzung in die Praxis wird abgerundet durch die Darstellung des dem Körper inne wohnenden Gefährdungspotentials im Sinne eines problematischen Körperverhältnisses am Beispiel eines kreativ-zeitgenössischen Tanz- und Improvisationsangebots im Kontext der Sozialen Arbeit mit Langzeitstraftätern in der Justizvollzugsanstalt, sowie am Beispiel der Arbeit mit Menschen mit Adipositas und im Kontext von Gewaltpräventionsforschung.

Den Abschluss des Buches bildet das Thema Körper bzw. leibliche Reflexivität aus der Perspektive der Organisationsberatung. Stefan Schache eröffnet in seinem Beitrag das Potential einer Organisationskultur, die sich in ihrem Selbstverständnis hin zu einer Inklusionskultur entwickelt und sich dem Leib in seiner grundlegenden Bedeutung und in seinem Potential für die Beratung- und Begleitung verstärkt zuwendet.

Das vorliegende Buch liefert einen wichtigen und gelungenen Beitrag zur Sensibilisierung und Problematisierung der unzureichenden Wahrnehmung und Integration körperlicher Dimensionen im Kontext Sozialer Arbeit. Die einzelnen Beiträge illustrieren anschaulich und differenziert die Vielfalt der Perspektiven auf den Körper in Abhängigkeit von Zielgruppen, Settings und zugrundliegendem Gefährdungspotential. Im Vordergrund steht ein theoretisches und praktisches Verständnis vom Körper, welches ihn, jenseits von einseitiger Biologisierung, in einen historisch entstandenen, sozialen und gesellschaftlichen Zusammenhang einbettet und ihn im Hinblick auf sein Potential zur ganzheitlichen Förderung von Ressourcen sowie zur Initiierung von Bildungs- und Identitätsentwicklungsprozessen würdigt. In ihrer besonderen Relevanz für das pädagogische Handeln ist hier insbesondere der Beitrag von Huster und Schütte zur Bedeutung der Vorprägung durch den Habitus und der Möglichkeit zur Veränderung des Habitus über die reflexive Förderung des sogenannten physischen Kapitals, zu nennen. Bezogen auf das pädagogische Handeln verweist auch Marie-Luise Hünerbein, mit ihrer Sensibilisierung für den weitreichenden Einfluss körperlicher Präsenz in Beratungs- und Begleitungsprozessen, auf den grundlegenden Zusammenhang von Selbstreflexion und Körper, hier bezogen auf das persönliche Wachstum und die professionelle Weiterentwicklung. Nicht zuletzt verdeutlicht die Vielfalt der vorgestellten Projekte am Beispiel von Theaterkunst, zirzensischen Künsten, kreativem Tanz und Improvisationen und die Schilderung der sich hier vollziehenden Entwicklungen, das Ressourcenpotential von Angeboten, die stärker körperliche sowie performative und ästhetische Elemente in den Fokus nehmen.

Wendler und Husters Herausgeberband nimmt sich Vernachlässigung des Körpers in der Sozialen Arbeit an und problematisiert diese, indem er die grundlegende soziale Einbettung des Körpers im Wechselverhältnis $\mathrm{zu}$ kognitiven und emotionalen 
Dimensionen, insbesondere im Kontext der Erziehungsarbeit mit Kindern und Jugendlichen, hervorhebt. Es ist vor diesem Hintergrund nur konsequent seitens der Autorinnen und Autoren, die stärkere Einbeziehung des Körpers nicht nur in der Praxis, sondern gerade und besonders im Kontext von Wissenschaft und Ausbildung zu fordern.

Indes erfolgt die Auseinandersetzung mit körperbezogenen Aspekten zu stark fokussiert auf Bewegung, Performanz, körperlicher Aktivität bzw. Sport. Zu wenig finden sich, jenseits von Ressourcen und Potential, Thematisierungen eben jenes körperlichen Phänomens, jener Körperpraxis, die für die Soziale Arbeit doch so offensichtlich konstitutiv sind, wie der widerständige Körper, der aus dem Rahmen fällt. Die Forderung nach Angeboten, die stärker zielorientiert den Körper in den Blick nehmen, ist zwar notwendig, bildet jedoch nur unzureichend die pädagogische Realität in der Lebenswelt der Adressatinnen und Adressaten Sozialer Arbeit, insbesondere im Kontext von Gesundheit und Krankheit, ab. Deren problematischer Umgang mit ihrem Körper, die Verweigerung von Pflege und Aufmerksamkeit dem Körper gegenüber, die häufig extreme Vernachlässigung, Verletzung oder Zur-Schau-Stellung des Körpers, sind Phänomene denen mit Angeboten nur sehr begrenzt begegnet werden kann.

Um nachhaltig wirken zu können, muss es darüber hinaus um die Entwicklung eines Verstehenszugangs zum Körper gehen, der ihn, jenseits von Funktionalisierung und Zielorientierung, als Teil eines Beziehungsraumes anerkennt, und damit die Grundlage schafft, Adressatinnen und Adressaten zu einem reflexiven Verhältnis zu ihrem eigenen Körper zu verhelfen. Um dies zu realisieren, bedarf es eines sozialtheoretischen und psychodynamischen Verständnisses vom Körper, welches ihn als grundlegend für unser Subjektsein, unser So-Geworden-Sein und unser Soziales-Sein begreift und ihn damit als zentrale Sinn-Kategorie in den Fokus professionellen Handelns rückt. 